Gynäk. Rdsch. 1986;26(suppl. 2):I-XII

\title{
Contents, Vol. 26, Supplement 2, 1986
}

Gitsch, E.: Begrüßungund Eröffnung der Tagung 1

1. Hauptthema: Die Geburtsüberwachung im Rah men einer verantwortungsbewußtenGeburtshilfe

Hauptreferat

Hickl, E.: Die Geburtsüberwachung im Rahmen einer verantwortungsvollen Ge-

burtshilfe 5

Koreferate

Sarembe, B.: Die Struktur der Schwangeren- und Geburtsüberwachung in der DDR 15 Bayer,

H.: Klinische Gesichtspunkte bei der Uberwachung von Schwangerschaften

und Geburten 22

Rosenkranz, A.; Fürnkranz, H.: Gegenüberstellung der kontinuierlich überwachten

Geburt zur nicht überwachten Geburt im Hinblick auf Mortalität und Morbi-

ditätdes Kindes $\quad 28$

Statement

Kratochwil, A.: Sicherheit des diagnostischen Einsatzes von Ultraschall in der

Schwangerschaft $\quad 32$

Mitteilungen zum 1. Hauptthema

Bichler, A.: Geburtshilfe heute: Forschung, Lehre, Praxis 36

Beck, A.: Zur perinatalen Mortalität in Wien 1984 und 1985 - Ergebnisse der Einzel-

fallanalyse $\quad 40$

Schmid, R.; Bauer, E.; Floder, F.: Management und Fetal Outcome bei Beckenendlagen am KH Mistelbach $\quad 43$

Inhalt IV

Ringler, M.: Psychologische Aspekte der Uberwachung der fetalen Herzfrequenz: Zur Diskussion zwischen schulmedizinischen und «alternativen» Betreuungsstrategien 45

Gerstner, G.J.; Gredler, B.: Zur Diskussion um die «sanfte Geburt»

Schaller, A.; Vutuc, C; Bartsch, F.; Bodingbauer, G.; Bodingbauer, J.; Fiedler, T.;

Fröhlich, H.; Golob, E.; Gottschall, J.; Gruber, L.; Hoffelner, F.; Hoser, G.;

Kölblinger, A.; Konrad, E.; Krupitz, H.; Marberger, F.; Moser, A.; Moser, W.;

Nagl, F.; Seidl, A.; Stöger, H.; Schuhmeier, V.; Winkler-Ramharter, R.:

Häufigkeit und Inzidenz von angeborenen Fehlbildungen. Modellstudie -

Oberösterreich 50

Freie Vorträge: Geburtshilfe: GebuГt und Neugeborenes

Beck, A.; Vutuc, C: 10 Jahre Müttersterblichkeitsuntersuchung in Österreich .... 52

Rudelstorfer, R.; Gruber, W.: Hausgeburtshilfe in den Niederlanden

Endler, M.: Die Hausgeburt in Wien in den letzten 25 Jahren 57

Mutke, H.G.: Die «natürliche» Geburt, die Kreißsaaltechnik und das geburtsgeschädigteKind 
Rosanelli, K.; Ritschl, E.: Einfluß von Erstversorgung und Transport auf das Schicksal schwer asphyktischer, reifer Neugeborener 61

Gmoser, G.; Pavelka, R.: Nachuntersuchung beatmeter und nicht beatmeter Neu geborener miteinem Geburtsgewicht unter $2500 \mathrm{~g} \quad 64$

Schmid, R.: Computergestützte perinatologische Einzelfallanalyse in Niederösterreich. Erfahrungsbericht über das Jahr 198566

Gitsch, G.; Schatten, C: Die Klavikularfraktur als Geburtstrauma 69

Langer, M.; Ringler, M.; Sagl, R.; Reinold, E.: Telemetrische oder stationäre Geburtsüberwachung: Unterschiede im Geburtserleben 71

Pavelka, R.; Gmoser, G.: Der richtige Abnabelungszeitpunkt? Blutgasanalysen, Hämatokrit 75

Rudelstorfer, R.; Gruber, W.; Simbruner, G.; Janisch, H.: Der Wärmefluß vom feta len Scalp zur Vorhersage der Lebensfrische des Neugeborenen 78

Simbruner, G.; Weninger, M.; Entacher, U.; Rudelstorfer, R.: Der Wärmefluß vom Kopf Neugeborener mit abnormem cerebralem Metabolismus und Kreislauf . 80 Simbruner, G.; Weninger, M; Entacher, U.; Rudelstorfer, R.: Der Wärmefluß vom Kopf Neugeborener und seine Abhängigkeit von der Perfusion der Kopfhaut Ralph, G.; Lang, P.; Lichtenegger, W.; Kömetter, R.: Zwillingsgeburten 85 Bartussek, G.; Spernol, R.; Szalay, S.: Schwangerschaftsdauer und Geburtsgewichte bei Zwillingsschwangerschaften 88

Wibiral, G.; Eppel, W.; Reichel, R.: Schwangerschaftsdauer und Geburtsgewicht bei Zwillingsschwangerschaften mit oder ohne prophylaktische Cerclage eine Vergleichsstudie 90

Ralph, G.; Lichtenegger, W.; Lang, P.; Mayer, H.O.: Sektio beim zweiten Zwilling

Freie Vorträge: Geburtshilfe: Schwangerschaft

Stummvoll, W.; Prestel, A.; Wiebogen, L.; Nagl, F.: Gibt es das Problem des sogenannten CTG-Versagers? 97

Inhalt

IX

Czerwenka, K.; Böhm, R.; Sedlak, O.; Fröch, A.: Primäres Karzinoid des Ovars: Immunhistochemie und Elektronenmikroskopie - ein Vergleich 289 Auner, J.; Lahousen, M.; Ralph, G.; Mayer, H.O.; Beaufort, F.: Phosphohexoseisomerase als Überwachungsparameter bei Patientinnen mit Ovarialkarzinom . . 290 Czerwenka, K.; Kremser, K.; Retzek, H.: Glykoproteinmuster von ovarialen (neoplastischen) Zysteninhalten und -epithelien mittels der «Lektinblotting»Methode 293

Reichel, R.; Sevelda, P.; Haider, F.; Salzer, H.; Knapp, W.; Ganzinger, U.: Immunpharmakologische Untersuchungen mit Äthylenbisdithioäthanol (Sandoz SubstanzNr. ADA 202-718) bei Patientinnen mit Ovarialkarzinom 295 Sevelda, P.; Genger, H.; Czerwenka, K.; Spona, J.: CA-125 - Ein Tumormarker für Früherkennung maligner epithelialer Ovarialkarzinome? 297

Genger, H.; Sevelda, P.; Salzer, H.; Spona, J.: Serologische Tumormarkerbestimmungbeim Ovarialkarzinom: CA-125 versus CA-153 299

Neunteufel, W.; Bieglmayer, C; Kölbl, H.; Schieder, K.; Breitenecker, G.: Der Gehalt der Tumormarker CA 125, CA 19-9 und CEA in Gewebsschnitt und Serum von Patienten mit Ovarialtumoren 301 
Lahousen, M.; Stettner, H.; Pickel, H.; Urdl, W.; Pürstner, W.: Tumormarker versus Second-look-Operation beim Ovarialkarzinom 304

Kölbl, H.; Schieder, K.; Nowotny, C; Breitenecker, G.; Neunteufel, W.; Csaicsich, P.; Szepesi, T.; Janisch, H.: Der Einsatz der Second-look Operation in der Beurteilung einer adjuvanten Chemotherapie (Adriamycin/Cisplatin) bei fortgeschrittenem Ovarialkarzinom 305

Sevelda, P.; Genger, H.; Salzer, H.; Kucera, H.: Die Prognose des inoperablen Ovarialkarzinoms in Abhängigkeit von der Nachbehandlung 308

Schieder, K.; Kölbl, H.; Bieglmayer, C; Neunteufel, W.; Breitenecker, G.: Heterogenität von Steroidrezeptoren bei Ovarial-und Endometriumkarzinom .... 310 Knogler, W.; Karlic, H.; Huber, J.C.; Söregi, G.; Kucera, H.: Vergleichende Untersuchung der Wirkung verschiedener Progestine beim Endometriumkarzinom im Hinblick aufeinen strahlensensibilisierenden Effekt in vitro 312 Gredler, B.; Gerstner, G.J.: Regionale Probleme des Zervixkarzinom-Screenings in Österreich 315

Reichel, R.; Husslein, P.; Kucera, H.; Sinzinger, H.: Plättchenfunktion unter der Strahlentherapie bei Patientinnen mit Zervixkarzinom 317

Lahousen, M.; Haas, J.; Pickel, H.: Adjuvante Polychemotherapie beim radikal operierten Zervixkarzinom 321

Fuith, L.C.; Daxenbichler, G.; Dapunt, O.: Klinische Relevanz des tumorassoziierten Antigens SCC bei Plattenepithelkarzinomen der Zervix uteri 322

Autorenregister

327

Inhalt

$\mathrm{V}$

Pleban, R.: Perinatales Management an einer kleinen Wiener Geburtshilflichen Abteilung 99

Leodolter, S.; Bali, C;Irsigler, K.; Pilz, I.; Rost, I.: EinflußderGlukosetoleranzinder

Schwangerschaft auf neonatale Pathologie 102

Rost, I.; Fuchs, G.; Leodolter, S.; Pilz, I.: Geburtshilfliche und kindliche Daten einer prospektiven Wiener Studie zur Erfassung des gestationalen Diabetes 105

Feiks, A.; Nowotny, C; Dadak, C; Czerwenka-Horworka, K.; Waldhäusl, W.: Nahenormoglykämische Insulinsubstitution bei IDDM und Schwangerschaft .... 107 Schurz, A.R.; Fritzsche, H.: Die Plazentadurchströmung bei gleichzeitiger BetaStimulation mit Hexoprenalin und Beta-1 -Blockade mit Celiprolol 109 Lechner, W.; Heim, K; Zech, J.; Daxenbichler, G.; Marth, C: Diagnose der nutritiven Plazentainsuffizienz durch Östriolbestimmung im Speichel 111 Feiks, A.; Nowotny, C; Gruber, W.; Preisinger, E.; Trnavsky, G.; Janisch, H.: Rheographische Untersuchung der peripheren Haemodynamik in normaler und pathologischer Schwangerschaft 114 Skodler, W.D.; Philipp, K.; Sagl, R.; Pateisky, N.; Reinold, E.: Der Einsatz von gepulstem Doppler-Ultraschall zur Diagnose der Plazentainsuffizienz

Skodler, W.D.; Philipp, K.; Pateisky, N.; Brehm, R.: Messung der uteroplazentaren Durchblutung unter antihypertensiver Therapie mit gepulstem Doppler-Ultra schall 118

Dörfler, V.; Fenzl, E.; Wais, G.; Schuster, K.: Ultraschall in der pränatalen Diagno- 
stik fetaler Mißbildungen. EineDarstellunganhand von Fallberichten

Pateisky, N.; Egarter, C; Lapin, A.; Schurz, B.; Gabl, F.: C-reaktives Protein beim vorzeitigen Blasensprung 122

Feiks, A.; Dadak, C; Lasnik, E.: Thrombozytopenie und Hepatopathie bei Präeklampsie 124

Neunteufel, W.; Deutinger, J.; Reinthaller, A.; Csaicsich, P.; Müller-Tyl, E.: Bestimmung von $\mathrm{pCC}^{1 / 8} \mathrm{p}, 2$ und $\mathrm{pH}$ in Fruchtwasser und Nabelschnurblut 126

Schurz, B.; Eppel, W.; Egarter, C; Knogler, W.; Huber, J.C: Nabelschnurhormonbestimmungen unmittelbar post partum 128

Dadak, C; Deutinger, J.; Reinthaller, A.; Neumark, J.; Hoche, C: Arachidonsäuremetaboliten in Nabelschnurgefaßen von rauchenden und nichtrauchenden

Frauen 131

Enzelsberger, FL; Knogler, W.; Sevelda, P.; Huber, J.C; Legenstein, J.: Phospholipase A2-Konzentration während der Geburt 133

Reichel, R.; Bruck, P.; Eppel, W.; Widhalm, K.: Lipoproteine und Apoproteine wäh rend der Schwangerschaft - erste Ergebnisse einer Longitudinalstudie 135

Vytiska-Binstorfer, E.; Genger, H.; Huber, J.C: Amniale AFP-Konzentration in

Schwangerschaften mit numerischen Chromosomenaberrationen 138

Bieglmayer, C; Fischl, F.: Rasche Bestimmungsmethoden für LH aus Harnproben . 139

Motter, W.; Ralph, G.; Lichtenegger, W.: Überwachung bei Geburtseinleitung durch

intravaginale Applikation von Prostaglandin $7 / 8$-Tabletten 142

Reichel, R.; Husslein, P.; Göschen, K.; Rasche, M.; Sinzinger, H.: Plasma-Prosta-

glandin-E2-Metabolit-Konzentration nach lokaler Applikation von Prostaglan-

$\operatorname{din}^{7} / 8$ zur Geburtseinleitung 144

Egarter, C; Kofler, E.; Husslein, P.: Retrospektive Analyse von 2149 Geburtsein-

leitungen durch intravaginale Prostaglandin-E2-Tablettenapplikation $\quad 146$

Inhalt

VI

Gredler, B.; Gerstner, G.J.: 1st die regelmäßige vaginale Untersuchung heute integraler Bestandteil der Schwangerenvorsorge in Österreich? 148

Neumann, H.: 1st eine intensive Schwangerenbetreuung (skand. Modell) in Österreich möglich?

Und wenn, mit welchen Ergebnissen kann gerechnet werden? . 150

Neumann, H.: Was wird aus einer Schwangerschaft? Erste Ergebnisse aus einem

Jahr Praxisalltag 152

Wiebogen, L.; Nagl, F.; Prestel, A.; Stummvoll, W.: Schwangerschaft und Geburt bei

Eisenmengersyndrom 154

Bieglmayer, C; Feiks, A.; Neunteufel, W.; Rudelstorfer, R.: Serumkonzentrationen

der Basalmembrankomponente Laminin bei Schwangerschaften 156

Kraus, T.; Beck, A.: Geburtshilfliche Daten von Adoptionskindern 158

2. Hauptthema: Physiologie und Pathologie des Zyklus

Beitrag nicht eingelangt!

Koreferate

Huber, J.C.: Neuroendokrinologie des Menstruationszyklus 161

Tscherne, G.: Hyperprolaktinämie und Ovarialfunktion 165

Tabarelli, M.; Daxenbichler, G.; Penz, A.; Dapunt, O.: Die Bedeutung der Androgenämie für den weiblichen Genitalzyklus 171 
Freie Vorträge: In-vitro-Fertilisierung

Szalay, S.; Spernol, R.; Nachtigall, M.: Zwei Jahre in vitro Fertilisíerungsprogramm in Klagenfurt. Erfahrungen und Ergebnisse 179

Spernol, R.; Szalay, S.; Nachtigall, M.: Verbesserung der Schwangerschaftsrate und Senkung der Abortusfrequenz nach IVF-ET durch ein Östrogen-Gestagengemisch 181

Csaicsich, P.; Reinthaller, A.; Deutinger, J.; Riss, P.; Müller-Tyl, E.; Fischl, F.; Bieglmayer, C; Janisch, H.: Hormonparameter und Fertilisierung menschlicher Eizellen in Abhängigkeit vom Serum-Prolaktin-Spiegel in stimulierten Zyklen 183

Freude, G.; Leodolter, S.; Mossig, H.; Gasser, G.: Intratubarer Gametentransfer eine sinnvolle Ergänzung zu verschiedenen Methoden der Sterilitätsbehandlung? 185

Karlic, H.; Knogler, W.; Söregi, G.; Huber, J.C.; Egarter, C; Schurz, B.; Metka, M.: Bedeutung bakteriologischer Befunde im Sperma für die in-vitro-Fertilisation 188 Krause, P.; Huter, O.; Karpellus, E.; Kofler, J.; Tabarelli, M.: Keimverteilung und Empfindlichkeitsmuster in Samen asymptomatischer Manner steriler Ehepaare 191

Frei Vorträge: Endokrinologie und Sterilität

Schurz, B.; Schmidt, J.B.; Metka, M.; Huber, J.C.: Zur klinischen Bedeutung des TRH-Tests bei der Sterilitätsabklärung und beim androgenetischen Effluvium 195 Inhalt

VII

Urdl, W.; Desoye, G.; Pusch, H.H.: Zyklusstörungen bei Adipositas und polyzystischem Ovar-Syndrom. Die Östron-Androstendion-Ratio als differentialdiagnostisches Kriterium 198

Tabarelli, M.; Daxenbichler, G.; Karpellus, E.; Huter, O.; Pallua, A.; Mayr, U.: Thyreotropin Releasing Hormon Stimulation von Prolaktin: Bedeutung für die Diagnostik bei Patienten mit Hyperprolaktinämie 201

Enzelsberger, H.; Metka, M.; Schurz, B.; Binder, K.; Knogler, W.; Huber, J.C.: Vergleich von TRH- und MCP-Test bei latenter Hypeprolaktinämie 204 Knogler, W.; Lapin, A.; Karlic, H.; Egarter, C; Schurz, B.; Söregi, G.; Huber, J.C.; Gabl, F.: Analyse qualitativer Veränderungen von Polypeptiden des Endometriums im menstruellen Zyklus - eine vergleichende Studie bei fertilen Frauen und Patientinnen mit Lutealinsuffizienz 206 Kowatsch, A.W.; Tscherne, G.; Urdl, W.: Substitutionstherapie nach der prämaturen Menopause 208

Metka, M.; Enzelsberger, H.; Kurz, C.; Schurz, B.; Huber, J.C.; Knogler, W.; Langer, M.; Heytmanek, G.: Osteoporosefrüherkennung im Rahmen des klimakterischen Syndroms 211

Karpellus, E.; Tabarelli, M.; Huter, O.; Krause, P.: Verteilung der Therapieerfolge in Abhängigkeit von den therapeutischen und diagnostischen Maßnahmen bei sterilen Paaren 213

Boschitsch, E.; Leodolter, S.: Die Computerdokumentation in der Sterilitätsbehandlung 216

Freie Vorträge: Gynäkologie

Grünberger, W.: Zur Diagnose der chronischen Tubargravidität 
Schausberger, L.; Golob, E.: Sonographische Diagnostik der Extrauteringravidität . 221 Albrich, W.; Lentsch, P.; D’Acunto, A.: Zum Wandel der operativen Behandlung der Tubargravidität unter Berücksichtigung mikrochirurgischer Operationsmethoden 223

Pavelka, R.: Erfahrungen mit der suprapubischen Harnableitung bei vaginalen Inkontinenzoperationen 225

Rammer, E.; Friedrich, F.; Pollmann, B.: Verminderung von Harnwegsinfekten nach gynäkologischen Operationen durch Verwendung von suprapubischen Blasenkathetern 227

Kosian, K; Feiks, A.; Gruber, W.: Suprapubische Harnableitung nach vorderer Kolporrhaphie $\quad 230$

Riss, P.; Kölbl, H.; Janisch, H.: Urodynamische und morphologische Ergebnisse nach vorderer Levatorplastik 232

Leodolter, S.; Marhold,W.: Einseltener Fall von Endometriose 234

Kapellus, E.; Tabarelli, M.; Dapunt, O.: Die Endometriosebehandlung mit dem LH-RH-Agonisten Buserelin 236

Reichel, R.; Philipp, K.; Spona, J.; Gitsch, E.: Wirkung des GnRH-Agonisten Buserelin auf die Serum-Östradiol-Konzentration und auf Ovulation und Menstruation - Vorläufige Ergebnisse einer Studie bei Patientinnen mit Endo metriose 239

Inhalt

VIII

Hautmann, M.; Becker, H.; Hötzinger, H.: Möglichkeiten der Vaginosonographie mit dem Linear- und Sektorscanner zur Ergänzung der transvesikalen Ultraschalluntersuchung 241

Gerstner, G.J.; Schramek, P.: Ultraschall - gezielte, perkutane Punktion und Drai nage - eine neue Methode zur Behandlung von postoperativen Lymphzysten oder Abszessen 243

Kubista, E.; Gitsch, E.; Simmen, U.; Dreher, K.: Die Klammernahttechnik bei gynäkologischen Operationen 245

Krause, P.; Bichler, A.: Klinische Erfahrungen mit der lupenoptischen Q3/4-LaserBehandlung von Kondylomen 247

Knogler, W.; Schurz, B.; Salzer, H.; Husslein, P.: Präoperative Zervixdilatation vor Curettagen bei Nichtschwangeren 250

Vavra, N.; Sevelda, P.; Kucera, H.; Grünberger, W.: Zur Frage der Operabilität im Senium 252

Auner, J.; Ralph, G.; Lahousen, M.; Mayer, H.O.; Beaufort, F.: Die klinische Bedeutung des C-reaktiven Proteins in der Adnexitisdiagnostik 254

Fischl, F.; Kölbl, H.; Wolf, G.: Der Einsatz von Econazol-Nitrat in der prophylaktischen Anwendung vor Hysterosalpingographien 260

Pfersmann, C; Riegler, R.; Bunzel, B.; Dadak, C: Therapie der Mastodynie durch Akupunktur 262

Knogler, W.; Pickard, E.; Huber, J.C.: Take-me - Ein antikonzeptiver DiaphragmaSchwamm 263

Müller, G.; Gerstner, G.J.: Erfahrungen mit lyophilisierten Döderlein-Bakterien bei der Therapie des unspezifischen Fluor vaginalis 265 
Stadler, A.; Rutar, M.; Wolfesberger, B.; Szalay, S.: Inzidenz der Chlamydieninfektionen nach Risikogruppen 267

Scheer, I.: Der Nächste bitte! (Das Gespräch mit den Patienten) 269

Freie Vorträge: Onkologie

Arns, H.H.: Karzinom des äußeren Genitales und der Brust in der Praxis: Karzino-

genese, Frühdiagnose, Prophylaxe, Resultate 271

Bieglmayer, C; Szepesi, T.: Tumorwachstum und Laminin-Serumkonzentrationen

beim Mammakarzinom 274

Philipp, K.; Pateisky, N.; Enzelsberger, H.; Kubista, E.; Schatten, C: Stellenwert der

Immunlymphszintigraphie beim Mammakarzinom 1/8 277

Langer, M.; Kubista, E.; Spona, J.: Androgenspiegel und Hormonrezeptoren bei

Frauen mit Mammakarzinom und gutartigen Brusterkrankungen 278

Marth, C.; Daxenbichler, G.; Mayer, I.; Schmid, K.; Dapunt, O.: Interferon Gamma

hindert Mammakarzinomzellen an der Invasion in das Bindegewebe 281

Bartl, W. et al.: Protoadjuvante Chemotherapie versus Radiotherapie beim Brust-

krebs im Stadium T 3/4

282

Szepesi, T.; Bieglmayer, C: CA 15.3 und CEA bei der Therapiekontrolle und Nach-

sorge von Mammakarzinomen 284

Gerstner, G.J.; Ludwig, H.: Burkitt-Lymphome beider Ovarien bei einer 18jährigen

Türkin 287

ditorial

Wahrscheinlich lag es am Interesse an den Themen, die am Pro-gramm der Jahrestagung der

Österreichischen Gesellschaft für Gynäkolo-gie und Geburtshilfe standen, daß ein Rekord der

Besucherzahl an österreichischen Frauenärzten zu verzeichnen war. Das erste Hauptthema war von brennender Aktualität: „Die Geburtsüberwachung im Rahmen einer

verantwortungsbewußten Geburtshilfe". Dieses Thema wurde nicht zuletzt deswegen gewählt, weil, nach den Erkenntnissen und Fortschritte gerade auf diesen Gebieten der Medizin, vereinzelt Stimmen laut wurden, die diese wieder in Frage stellten und darauf verzichten wollen. Wie in der Eröffnungsrede des Präsidenten ausgeführt, wurden dazu Referenten aus alien deutschsprachigen Ländern eingeladen um über die Erfahrungen und Ansichten aus ihren Abteilungen und Kliniken zu berichten. Zusätz-lich wurden zahlreiche Vorträge aus vielen Abteilungen Österreichs zu diesem Thema angemeldet und gehalten; bedauerlicherweise jedoch kein Vortrag von jenen, die die nun schon allgemein in die Routine aufgenom-menen Uberwachungsmethoden in Frage stellen oder gar ablehnen. Es ware eine gute Möglichkeit gewesen, ihre Argumente und Erfahrungen vorzustellen und nicht nur in den Massenmedien. Auch die Laienpresse, die sich verschiedentlich dafür stark gemacht hat und sehr oft recht einseitig darüber berichtet hat, wurde zwar eingeladen; bedauerlicherweise kam es jedoch in dieser Hinsicht zu keiner Reaktion. Das zweite Hauptthema war der Endokrinologie gewidmet, ein be-sonderer Aspekt war in diesem Zusammenhang die in-vitro-Fertilisie-rung. Auch auf diesen Bereichen sind neue Erkenntnisse und Fortschritte erzielt worden, die in mehreren Referaten und Vorträgen vorgestellt wurden.

Die große Zahl der „Freien Vorträge” zeigt, daß auch in alien anderen Bereichen der Gynäkologie und Geburtshilfe viel und mit Erfolg gearbeitet wird. Besonders beachtenswert ist es, daß dies nicht nur für die Universitätskliniken zutrifft, sondern auch für mittlere und kleinere Abteilungen in ganz Österreich.

Editorial 
XII

Auch in diesem Jahr ist es uns ein aufrichtiges Bedürfnis, den Sponsoren unseren Dank auszusprechen; mit ihrer Unterstützung für das Erscheinen des Bandes haben sie ihre Verbundenheit mit unserem Fach-gebiet neuerlich bewiesen.

Unser Dank gilt aber auch den Mitarbeitern des Verlages S. KARGER, Basel, sowie der Druckerei Ferdinand BERGER, Horn in Niederösterreich, für die gute Zusammenarbeit. E. Gitsch E. Reinold 